\title{
Cross-sectional study to evaluate the association between Vitamin D status and endothelial function in overweight and obese, post-menopausal women
}

\author{
A.M. Hussin ${ }^{1,2}$, J. Mathers ${ }^{1}$, T. Hill ${ }^{3}$ and M. Siervo ${ }^{1}$ \\ ${ }^{1}$ Human Nutrition Research Centre, Institute of Cellular Medicine, Newcastle University, Campus for Ageing and \\ Vitality, Newcastle upon Tyne NE4 5PL, United Kingdom, ${ }^{2}$ Institute of Medical Science Technology, Universiti Kuala \\ Lumpur, Kuala Lumpur, Malaysia and ${ }^{3}$ School of Agriculture, Food and Rural Development, Newcastle University, \\ Newcastle upon Tyne, United Kingdom
}

Cardiovascular diseases (CVDs) are a major public health concern and are associated with an increased risk for mortality and morbidity $^{(1)}$. Many studies suggest that low levels of vitamin D are associated with an increased risk of CVD ${ }^{(1,2)}$. This association may be explained by the link between vitamin D (25OHD) with pathways involved in the regulation of insulin signalling and endothelial function $(\mathrm{EF})^{(3)}$.

The aim of this cross-sectional study was to investigate the associations between plasma 25OHD concentration and EF (flow mediated dilation and pulse wave velocity). Eighty healthy (with no established medical diagnosis), non-smoking, overweight and obese, post-menopausal women with a body mass index (BMI) between 25.0 and $40.0 \mathrm{~kg} / \mathrm{m}^{2}$, and age range between 50 to 70 years old were recruited in the study. Plasma 25OHD was measured by using immunoassay. Basic anthropometric measurements, body composition, resting blood pressure, post-occlusive reactive hyperaemia, pulse wave velocity were obtained using standard protocols.

Approximately $37.5 \%$ of the participants were vitamin D sufficient ( $>50 \mathrm{nmol} / \mathrm{L})$. Majority of the participants $(41 \cdot 3 \%)$ were vitamin D insufficient $(25-50 \mathrm{nmol} / \mathrm{L})$ and $21 \cdot 3 \%$ were vitamin $\mathrm{D}$ deficient $(<25 \mathrm{nmol} / \mathrm{L})$.

\begin{tabular}{|c|c|c|c|}
\hline & $\begin{array}{l}\text { Without deficiency } \\
(50 \mathrm{nmol} / \mathrm{L} \text { and } \\
\text { above, } \mathrm{n}=30)\end{array}$ & $\begin{array}{c}\text { Deficiency } \\
\text { (less than } 50 \mathrm{nmol} / \mathrm{L} \text {, } \\
\mathrm{n}=50)\end{array}$ & p-value \\
\hline Age (y) & $61 \cdot 4 \pm 7 \cdot 0$ & $61 \cdot 0 \pm 5 \cdot 6$ & $<0.05$ \\
\hline Height $(\mathrm{cm})$ & $159 \cdot 4 \pm 6 \cdot 2$ & $160 \cdot 6 \pm 6 \cdot 4$ & $0 \cdot 96$ \\
\hline Weight (kg) & $77 \cdot 2 \pm 14 \cdot 4$ & $76 \cdot 1 \pm 13 \cdot 6$ & 0.82 \\
\hline BMI (kg/m2) & $30 \cdot 0 \pm 4 \cdot 9$ & $29 \cdot 4 \pm 4 \cdot 2$ & 0.23 \\
\hline $\mathrm{WC}(\mathrm{cm})$ & $96 \cdot 7 \pm 14 \cdot 0$ & $94.8 \pm 10 \cdot 9$ & 0.99 \\
\hline Fat Mass (kg) & $31.9 \pm 10 \cdot 4$ & $30 \cdot 8 \pm 10 \cdot 3$ & 0.69 \\
\hline $\mathrm{SBP}(\mathrm{mm} \mathrm{Hg})$ & $137 \cdot 3 \pm 22 \cdot 0$ & $130 \cdot 2 \pm 16 \cdot 4$ & $0 \cdot 20$ \\
\hline DBP $(\mathrm{mm} \mathrm{Hg})$ & $75 \cdot 2 \pm 11 \cdot 4$ & $74 \cdot 2 \pm 8 \cdot 2$ & $0 \cdot 36$ \\
\hline $\mathrm{PWV}(\mathrm{m} / \mathrm{s})$ & $-0 \cdot 8 \pm 0 \cdot 2$ & $-0 \cdot 7 \pm 0 \cdot 2$ & 0.69 \\
\hline RHI & $4 \cdot 3 \pm 2 \cdot 7$ & $5.8 \pm 11.5$ & 0.47 \\
\hline
\end{tabular}

Abbreviations: BMI, body mass index; WC, waist circumference; SBP, systolic blood pressure; DBP, diastolic blood pressure; PWV, pulse wave velocity; $\mathrm{RHI}$, reactive hyperaemia index

Among all subjects, PWV was negatively related to SBP $(\mathrm{r}=-0.412, \mathrm{P}<0 \cdot 01)$ and DBP $(\mathrm{r}=-0 \cdot 480, \mathrm{P}<0 \cdot 01)$. However, there are no significant relationship between arterial distensibility and vitamin D. The vitamin D deficient group had lower RHI value compared to vitamin D insufficient and vitamin D sufficient group. However, the differences are not significant.

In conclusion, the results of the present study did not support the hypothesis that serum 25OHD status is associated with vascular endothelial function among overweight and obese, post-menopausal women.

1. Lavie CJ, Dinicolantonio JJ, Milani RV, O'Keefe JH. Vitamin D and cardiovascular health. Circulation. 2013;128(22):2404-6.

2. Motiwala SR, Wang TJ. Vitamin D and cardiovascular risk. Current Hypertension Reports. 2012;14(3):209-18.

3. Kim JA, Montagnani M, Kwang KK, Quon MJ. Reciprocal relationships between insulin resistance and endothelial dysfunction: Molecular and pathophysiological mechanisms. Circulation. 2006;113(15):1888-904. 